\title{
Avaliação ultrassonográfica dos tendões flexores dos membros anteriores em equinos de vaquejada na microrregião do Alto Médio Gurgueia, Sul do Piauí, Brasil**
}

\section{Ultrasound evaluation of the flexor tendons of the forelimbs in horses in vaquejada horses in the Alto Médio Gurgueia, South of Piauí, Brazil}

\author{
Waddamis Tyson Nunes de Sousa, ${ }^{* *}$ Talia Fabrício Gonçalves, ${ }^{* *}$ Mikael Leandro Duarte de Lima Tolentino, ${ }^{* * \star}$ \\ Natanael Pereira da Silva Santos, ${ }^{* * *}$ Manoel Lopes da Silva Filho, ${ }^{* * * * *}$ Denise Cerqueira de Sousa ${ }^{* * * * *}$
}

\begin{abstract}
Resumo
Objetivou-se com este estudo demonstrar a influência do tipo de treinamento sobre as medidas dos tendões flexores palmares em equinos de vaquejada que correm à direita do boi na microrregião do Alto Médio Gurgueia, Sul do Piauí, Brasil. Foram utilizados oito animais, da raça quarto de milha e seus mestiços, de diferentes idades, peso e sexo, selecionados e questionados sobre o tempo que exerciam a atividade de vaquejada e o tipo de treinamento a qual eram submetidos, que refere-se a maneira como praticavam a prova correndo de que lado do boi, em uma prova de vaquejada no município de Cristino Castro, Sul do Piauí, Brasil, onde os participantes eram provenientes de diferentes haras da Microrregião do Alto Médio Gurgueia, Sul do Piauí, Brasil. Em cada animal foi realizada ultrassonografia das zonas IB, IIB e IIIB dos tendões flexores digital superficial (TFDS) e digital profundo (TFDP), obtendo-se as medidas dos diâmetros médio lateral (DML) e dorso palmar (DDP) através do plano transverso em cada uma das diferentes zonas. Os dados foram submetidos a análise estatística (ANOVA), utilizando-se o teste $t$ a nível de significância de $(p<0,05)$, observando-se que não houve diferença significativa entre as medidas avaliadas. De acordo com os resultados obtidos, cavalos atletas que correm a direita do boi não apresentam diferença significativa nos diâmetros médio lateral e dorso palmar dos tendões flexores entre os antímeros direito e esquerdo do membro torácico.
\end{abstract}

Palavras-chave: cavalo atleta, lesão, tendão, ultrassonografia.

\begin{abstract}
The objective of this study was to demonstrate the influence of the type of training on the measurements of the palmar flexor tendons in vaquejada horses that run to the right of the ox in the microregion of the Alto Médio Gurgueia, South of Piauí, Brazil. Eight animals of the quarter-mile breed and their crossbreed, of different ages, weight and sex, were selected and asked about the time they were engaged in the activity of the vaquejada and the type of training they were submitted to as they practiced running on which side of the ox, in a vaquejada test in the municipality of Cristino Castro, South of Piauí, Brazil, where the participants came from different farms of the microregion of the Alto Médio Gurgueia, South of Piauí, Brazil. The ultrasonography of the IB, IIB and IIIB zones of the superficial digital (TFDS) and deep digital flexor tendons (TFDP) were performed in each animal, obtaining the measurements of the lateral diameters (DML) and palmar dorsum (DDP) through the transverse plane in each of the different zones. Data were submitted to statistical analysis (ANOVA), using the $t$ test at the significance level of $(p<0.05)$, observing that there was no significant difference between the measures evaluated. According to the results, horses for vaquejada athletes do not present significant difference in the lateral diameters and palmar dorsum of the flexor tendons between the right and left thoracic limb antimeres.
\end{abstract}

Keywords: horse athlete, injury, tendon, ultrasonography.

\section{Introdução}

O Brasil possui um os maiores rebanhos mundiais de equinos, segundo o Ministério da Agricultura, Pecuária e Abastacimento (MAPA, 2016) a tropa nacional é superior a 5 milhões de cavalos inseridos em diversas atividades, a qual se incluem os cavalos atletas para vaquejada, atividade que foi criada há centenas de anos, considerada hoje uma tradição passada de geração a geração e que movimenta milhões na economia nacional (Lopes et al., 2009).

*Recebido em 7 de maio de 2019 e aceito em 30 de abril de 2021.

**Discente do curso de Bacharelado em Medicina Veterinária da Universidade Federal do Piauí do Campus Professora Cinobelina Elvas, Bom Jesus, Piauí, Brasil.

***Médico Veterinário do Hospital Veterinário Universitário da Universidade Federal do Piauí do Campus Professora Cinobelina Elvas, Bom Jesus, Piauí, Brasil. ****Docente do curso de Bacharelado em Zootecnia da Universidade Federal do Piauí do Campus Professora Cinobelina Elvas, Bom Jesus, Piauí, Brasil.

${ }^{* * * * *}$ Docente do curso de Bacharelado em Medicina Veterinária da Universidade Federal do Piauí do Campus Professora Cinobelina Elvas, Bom Jesus, Piauí, Brasil. denny_cerqueira@hotmail.com 
Durante as provas e treinamentos de vaquejada, além dos animais se exercitarem muito, os períodos de duração entre cada corrida são muito curtos e rápidos, sujeitando os animais a esforços físicos com alta intensidade, em um curto período de tempo ao limite máximo suportado pelo o organismo (Lopes et al., 2009), predispondo-os à lesões musculoesqueléticas, dentre as quais se destacam as tendinites (Ferraro et al., 2003; Meneses et al., 2014).

Os animais de vaquejada são selecionados para executar a atividade de tração do bovino com deslocamento do corpo em uma direção específica, direita ou esquerda. Aqueles que deslocam o corpo para o lado esquerdo são os cavalos de direita ou que correm a direita do boi. $\mathrm{E}$ os animais de esquerda ou que correm à esquerda do boi, aqueles que deslocam o corpo para o lado direito (Lyra et al., 2011).

Esse treinamento está diretamente ligado a um aumento na área de secção transversal (AST) em alguns tendões e regiões tendíneas, podendo ou não ser observadas alterações em suas propriedades mecânicas e estruturais, como o conteúdo de colágeno (Yamada et al., 2009).

Nos cavalos, os tendões flexores, não só flexionam o dígito durante a locomoção, mas também flexionam o membro, fazendo com que numa situação sob estresse, por exemplo, eles segurem a articulação do boleto quando o animal está parado, predispondoos a injúrias nessas estruturas (Bragante et al., 2010).

A inspeção e palpação são os meios mais utilizados para avaliar tendões e ligamentos, compondo o exame clínico do animal. Associado a isto, está a ultrassonografia, que permite uma avaliação mais precisa e precoce das lesões nas estruturas envolvidas, além de ser é um método eficiente na obtenção de medidas de biométricas, como podemos verificar no estudo de Pasin et al. (2001) que caracterizou ultrassonograficamente os tendões flexor digital superficial e flexor digital profundo e o ligamento acessório do dos membros anteriores em cavalos de diferentes tipos raciais, dentre esses cavalos de hipismo da raça Hanoveranos e seus mestiços, cavalos de Corrida Puros Sangue Inglês e Crioulos, onde observou ao comparar cortes transversais ultrassonográficos da articulação metacarpofalangeana em potros com dois a quatro anos, uma diminuição tanto da largura quanto da espessura do tendão do músculo flexor digital superficial e no estudo de Aristizábal et al. (2005) que determinou valores biométricos através da ultrassonografia dos tendões flexores da região metacarpiana em equinos da raça Mangalarga Marchador.

Sendo assim, objetivou-se com esse estudo demonstrar a influência do tipo de treinamento sobre as medidas dos tendões flexores palmares em equinos de vaquejada que correm à direita do boi na microrregião do Alto Médio Gurgueia, Sul do Piauí, Brasil.

\section{Material e métodos}

Para a realização desta pesquisa, todos os procedimentos experimentais foram realizados em conformidade com as diretrizes do Comitê de Ética em Experimentação Animal da Universidade Federal do Piauí, registro $n^{\circ} 434 / 18$.

Os dados foram coletados em uma prova de vaquejada no município de Cristino Castro, Sul do Piauí, Brasil, onde os participantes eram provenientes de diferentes haras da Microrregião do Alto Médio Gurgueia, Sul do Piauí, Brasil.

Foram selecionados oito cavalos da raça quarto de milha e seus mestiços, de diferentes idades, peso e sexo, que praticavam a prova correndo do lado direito do boi, não tenham sido submetidos ao uso de fármacos analgésicos ou anti-inflamatórios recentemente e nem apresentavam sinais de claudicação, sendo questionado sobre o tempo que exerciam as atividades e tipo de treinamento a qual eram submetidos, que refere-se a maneira como praticavam a prova correndo de que lado do boi.

Logo após realizou-se o preparo do membro para o exame ultrassonográfico, aplicando álcool a $70 \%$, seguida de uma espessa camada de gel acústico, retirando o ar presente entre o membro e o transdutor, evitando assim a formação de artefatos de imagem. Utilizou-se aparelho de ultrassom Samsung Medison ${ }^{\circledR}$ modelo SonoaceR3, obtendo-se imagens em modo-B, com transdutor linear na frequência de 7,5 MHz e com ajuste de ganho proximal de $100 \%$.

Os membros, tanto direito como esquerdo, foram avaliados em zonas pré-estabelecidas, sendo, de acordo com Genovese et. al. (1986), zonas IB (de 4-8 cm distal ao osso acessório do carpo), IIB (12-16 cm distal ao osso acessório do carpo), e IIIB (20-24 cm distal ao osso acessório do carpo), realizando-se mensurações do tendão flexor digital superficial e tendão flexor digital profundo, através do plano transverso, onde mensurou-se os diâmetros médio lateral e dorso palmar em cada uma das diferentes zonas IB, IIB e IIIB (Figuras 1, 2 e 3, respectivamente).

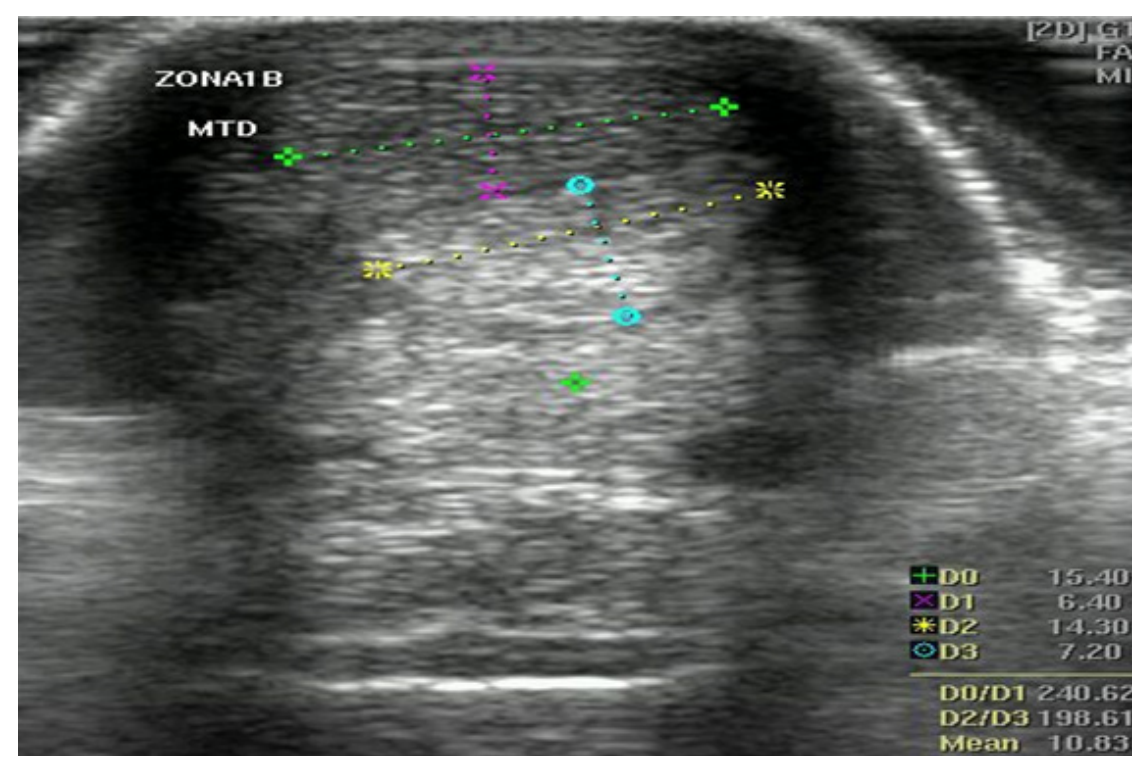

Figura 1: Imagem ultrassonográfica da região metacarpiana do membro direito de um cavalo, demonstrando as medidas dorso palmar e médio lateral do TFDS e do TFDP na zona IB. 


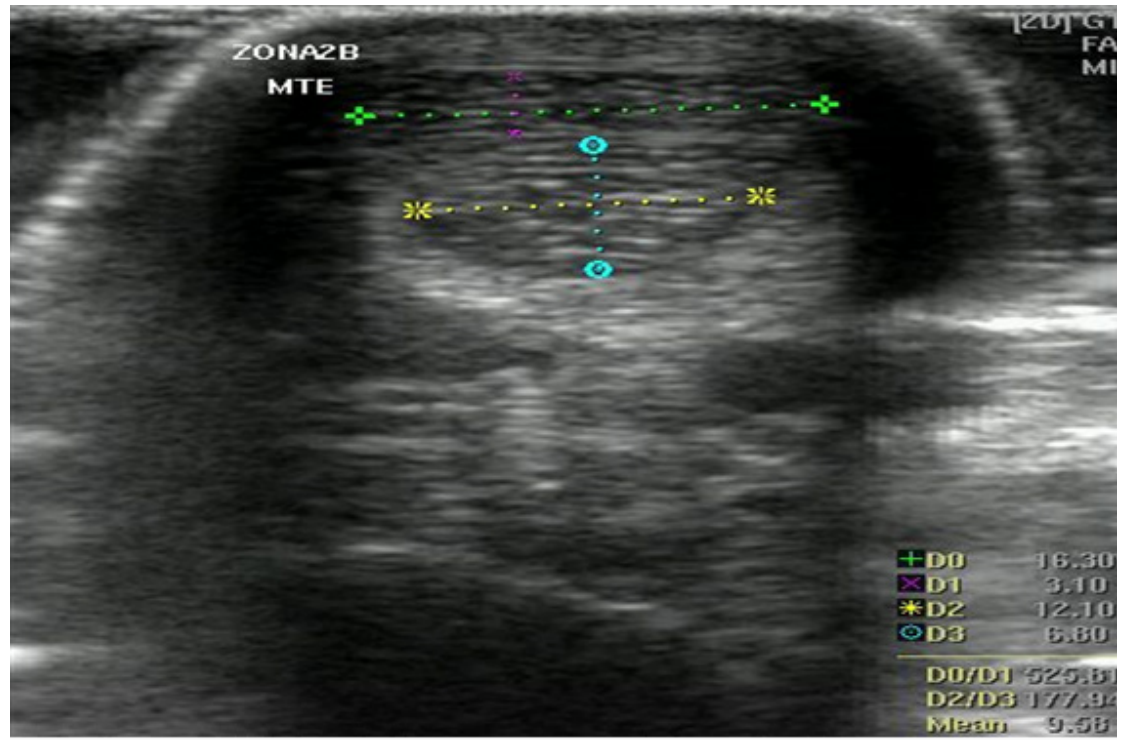

Figura 2: Imagem ultrassonográfica da região metacarpiana do membro esquerdo de um cavalo, demonstrando as medidas dorso palmar e médio lateral do TFDS e do TFDP na zona IIB.

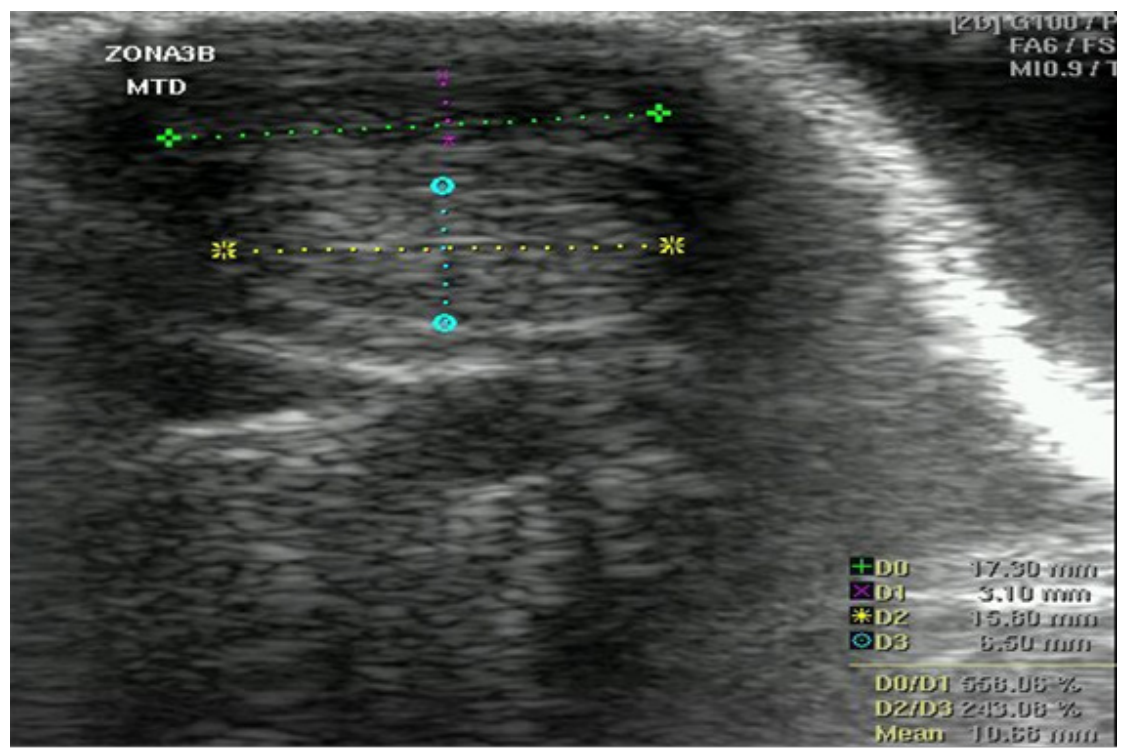

Figura 3. Imagem ultrassonográfica da região metacarpiana do membro direito de um cavalo, demonstrando as medidas dorso palmar e médio lateral do TFDS e do TFDP na zona IIIB.

Para análise estatística, foram consideradas as variáveis: tempo de treinamento, diâmetro do TFDS do membro direito, diâmetro do TFDP do membro direito, diâmetro do TFDS do membro esquerdo e diâmetro do TFDP do membro esquerdo, analisados nas três zonas (IB, IIB e IIIB). Para comparação foi realizada a análise de variância (ANOVA) utilizando o programa PROC GLM do software SAS ${ }^{\circledR}$ (versão University Edition), utilizando-se o teste $t$ a nível de significância de $5 \%(p<0,05)$.

\section{Resultados e discussão}

Não foram observadas diferenças significativas nos diâmetros médio lateral (MDL) e diâmetros dorso palmar (DDP) dos tendões flexores entre os antímeros direito e esquerdo do membro torácico dos equinos avaliados (Tabelas 1 e 2). 
Tabela 1: Dados das medidas dos diâmetros do tendão flexor digital superficial de cavalos de vaquejada, obtidos através do estudo ultrassonográfico e do tempo de treinamento a partir da aplicação de questionários aos proprietários

\begin{tabular}{c|c|c|c}
\hline \multirow{4}{*}{ MEMBROS } \\
\hline \multirow{2}{*}{$\begin{array}{c}\text { DIÂMETROS } \\
(\mathrm{mm})\end{array}$} & VARIAVEIS & DIREITO & ESQUERDO \\
\cline { 2 - 4 } & $\mathrm{DP}$ & $3,94^{\mathrm{a}}$ & $4,15^{\mathrm{a}}$ \\
\cline { 2 - 4 } & $\mathrm{ML}$ & $16,40^{\mathrm{a}}$ & $16,41^{\mathrm{a}}$ \\
\hline TEMPO DE TREINO & $1 \mathrm{a} 4$ & $10,37^{\mathrm{a}}$ & $10,33^{\mathrm{a}}$ \\
\cline { 2 - 4 }$($ ANOS) & $>4$ & $9,97^{\mathrm{a}}$ & $10,23^{\mathrm{a}}$ \\
\hline \multirow{2}{*}{ ZONAS } & $1 \mathrm{~B}$ & $9,79^{\mathrm{a}}$ & $9,68^{\mathrm{a}}$ \\
\cline { 2 - 4 } & $2 \mathrm{~B}$ & $9,29^{\mathrm{a}}$ & $9,96^{\mathrm{a}}$ \\
\cline { 2 - 4 } & $3 \mathrm{~B}$ & $11,44^{\mathrm{a}}$ & $11,19^{\mathrm{a}}$ \\
\hline
\end{tabular}

DP: dorso-palmar; ML: médio-lateral. Médias seguidas de mesma letra não diferem entre si pelo teste t. a $5 \%$.

Tabela 2: Dados das medidas dos diâmetros do tendão flexor digital profundo de cavalos de vaquejada, obtidos através do estudo ultrassonográfico e do tempo de treinamento a partir da aplicação de questionários aos proprietários

\begin{tabular}{c|c|c|c}
\hline \multirow{4}{*}{ MEMBROS } \\
\hline \multirow{2}{*}{$\begin{array}{c}\text { DIAMETROS } \\
(\mathrm{mm})\end{array}$} & VARIAVEIS & DIREITO & ESQUERDO \\
\cline { 2 - 4 } & $\mathrm{DP}$ & $7,13^{\mathrm{a}}$ & $6,9^{\mathrm{a}}$ \\
\cline { 2 - 4 } & $\mathrm{ML}$ & $14,81^{\mathrm{a}}$ & $14,82^{\mathrm{a}}$ \\
\hline TEMPO DE TREINO & $1 \mathrm{a} 4$ & $11,21^{\mathrm{a}}$ & $11,08^{\mathrm{a}}$ \\
\cline { 2 - 4 }$($ ANOS) & $>4$ & $10,73^{\mathrm{a}}$ & $10,64^{\mathrm{a}}$ \\
\hline \multirow{2}{*}{ ZONAS } & $1 \mathrm{~B}$ & $10,68^{\mathrm{a}}$ & $10,38^{\mathrm{a}}$ \\
\cline { 2 - 4 } & $2 \mathrm{~B}$ & $9,74^{\mathrm{a}}$ & $9,66^{\mathrm{a}}$ \\
\cline { 2 - 4 } & $3 \mathrm{~B}$ & $12,49^{\mathrm{a}}$ & $12,53^{\mathrm{a}}$ \\
\hline
\end{tabular}

DP: dorso-palmar; ML: médio-lateral. Médias seguidas de mesma letra não diferem entre si pelo teste t. a $5 \%$.

\section{Referências}

ARISTIZÁBAL F.A., SOUZA M.V., ARANZALES J.R.M.; RIBEIRO JUNIOR J.I. Valores biométricos obtidos por ultra-sonografia dos tendões flexores e ligamentos acessório inferior e suspensório da região metacárpica palmar de cavalos Mangalarga Marchador. Arquivo Brasileiro de Medicina Veterinária e Zootecnia, v. 57, n. 2, p.156-162, 2005.

BRAGANTE, T.; BRANCO, E.; BAILEIRO, J. C. C.; GOMES, T. L. S.; ZOPPA, A. L. V. Morfometria das principais estruturas que compõem a articulação metacarpofalangeana em equinos sem raça definida. Acta Veterinária Brasileira, v. 4, n. 4, p. 259-266, 2010.

FERRARO, G. C.; MORAES, J. R. E.; PEREIRA, G. T.; CAMARGO, M. H. B.; MORAES, F. R. Estudo morfológico de tendões flexores de equinos. Brazilian Journal of Veterinary Research and Animal Science, v. 40, n. 2, p. 117-125, 2003.

GENOVESE, R. L.; RANTANEN, N. W.; HAUSER, M. L.; SIMPSON, B. S. Diagnostic ultrasonography of equine limbs. Veterinary Clinics of North America Equine Practice, v. 2, p. 145226, 1996.
Nota-se que o DDP e o DML do membro esquerdo apresentamse numericamente maiores que do membro direito, porém eles não diferem entre si estatisticamente, em desacordo com o que foi observado por Lyra et.al. (2011), que ao analisarem também tendões de equinos de vaquejada obteve valores significativamente superiores no membro de apoio, quando comparados ao membro contralateral, em metade das zonas tendíneas dos membros anteriores dos cavalos avaliados, independentemente do lado que correm.

O fato de não ter apresentado diferenças significativas pode ser justificada por não haver esforço físico em uma única direção, eles realizam um esforço físico intenso, porém de curta duração durante as competições (Lopes, 2009), não sendo suficientes para sobrecarregar apenas um membro, e durantes os treinos fora da pista os movimentos não são repetitivos e nem na mesma direção, o que não seria suficiente para demonstrar diferença em apenas um dos membros.

Devemos levar em consideração que os fatores que favorecem as lesões físicas e psicológicas nos cavalos de vaquejada, muitas vezes não estão diretamente relacionados ao exercício, mas a fatores ambientais como poluição sonora, exposição à temperatura elevada, contato com outros animais desconhecidos, manejo alimentar inadequado, entre outros fatores (Lopes, 2009). Moffat et al. (2008) ao estudarem cavalos Puro Sangue Inglês, também não observaram influência do treinamento sobre o TFDS entre antímeros.

\section{Conclusão}

Os equinos atletas para vaquejada da raça quarto de milha e seus mestiços que recebem treinamento correndo à direita do boi não apresentam diferença significativa nos diâmetros médio lateral e diâmetros dorso palmar dos tendões flexores entre os antímeros direito e esquerdo dos membros torácicos.

LYRA, V. N.; MENDONÇA, L. B. R.; CAMARA, D. R. Avaliação ultrassonográfica de tendões flexores dos membros anteriores em equinos de vaquejada. Revista Brasileira de Ciências Agrárias, v. 6, n. 4, p. 717-721, 2011.

LOPES, K. R. F.; BATISTA, J. S.; DIAS, R. V. C.; SOTO-BLANCO, B. Influência das competições de vaquejada sobre os parâmetros indicadores de estresse em equinos. Ciência Animal Brasileira, v. 10, n. 2, p. 538-543, 2009.

MAPA. Ministério da Agricultura, Pecuária e Abastecimento. Revisão do Estudo do Complexo do Agronegócio do Cavalo. 2016. Disponível em: http://www.agricultura.gov.br/. Acesso em agosto de 2019.

MOFFAT, P. A.; FIRTH, E. C.; ROGERS, C. W.; SMITH, R K. W.; BARNEVELD, A.; GOODSHIP, A. E.; KAWCAK, C. E.; MCILWRAITH, C. W.; VAN WEEREN, P. R. The influence of exercise during growth on ultrasonographic parameters of the superficial digital flexor tendon of young thoroughbred horses. Equine Veterinary Journal, v. 40, n. 2, p. 136-140, 2008.

MENESES, A. C. A.; DA COSTA, M. D.; MARUCH, S.; MOREIRA, P. R.; NETO, T. M. Medidas lineares e angulares de animais da raça Quarto de Milha utilizados em uma prova de vaquejada. Revista Brasileira de Ciência Veterinária, v.21, n. 4, p.256-261, 2014. 
PASIN, M.; BRASS, K. E.; ROSAURO, A. C.; OLIVEIRA, F. G.; FIGUEIRÓ, G. M.; FIALHO, S. S.; SILVA, C. A. M. Caracterização ultrassonográfica dos tendões flexores em equinos: região metacarpiana. Arquivo Faculdade de Veterinária da UFRGS, v. 29, n. 2, p. 131-138, 2001.
YAMADA, A. L. M.; ALVES, A. L. G.; HUSSNI, C. A; NICOLETTI; L. M.; THOMASSIAN, A.; WATANABE, M. J.; CARNEIRO, R. Comparação de diferentes doses de colagenase em modelo de indução de tendinite para equinos: estudo clínico e ultrasonográfico. Ciência Rural, v. 39, n. 4, p. 1124-1130, 2009. 